\title{
Hiperhomocisteinemia como um fator de risco cardiovascular e seu controle por meio das vitaminas B6, B9 e B12
}

Jonathan Neves de Carvalho Alves

Discente do Curso de Nutrição.

Centro Universitário de Barra Mansa

e-mail: Jonathan.neves.alves@gmail.com

Fernando Antônio Cabral de Souza Júnior

Docente e Coordenador do Curso de Nutrição.

Centro Universitário de Barra Mansa

e-mail: nutrição@ubm.br

Jucimara Martins dos Santos

Docente do Curso de Nutrição.

Centro Universitário de Barra Mansa

e-mail: nutrição@ubm.br 


\section{Resumo}

A doença cardiovascular é a principal causa de morte em todo o mundo e a homocisteína é um de seus marcadores biológicos. Indivíduos com a doença de Parkinson, sob o tratamento com a L-dopa, têm níveis de homocisteina aumentados. $\mathrm{O}$ presente estudo visa revisar na literatura o mecanismo pelo qual as vitaminas B6, B9 e B12 reduzem a homocisteína sérica; bem como, apresentar a fisiologia da elevação da homocisteina, em parkinsonianos, gerado pelo metabolismo da L-dopa. Trata-se de uma revisão bibliográfica em bases de dados de saúde, utilizando artigos os quais foram publicados nos últimos 10 anos, livros e periódicos sobre o tema em questão. Indivíduos com a doença de Parkinson sob o tratamento exclusivo de L-dopa, têm maior probabilidade de desenvolverem hiperhomocisteinemia, a qual é um fator de risco cardiovascular. Tanto a prevenção quanto o tratamento estão relacionado com uma alimentação rica em vitaminas B6, B9 e B12, adquiridos em uma alimentação saudável e equilibrada.

Palavras-chave: homocisteína, doença cardiovascular, doença de Parkinson, vitaminas do complexo b.

\section{Abstract}

The cardiovascular disease is the leading cause of death worldwide and homocysteine is one of its biological markers. Individuals with Parkinson's disease, under treatment with L-dopa, have increased homocysteine levels. the present study aims to review in the literature the mechanism by which vitamins B6, B9 and B12 reduce serum homocysteine; as well as to present the physiology of elevation of homocysteine in parkinsonians generated by the metabolism of Ldopa. This is a bibliographical review in health databases, using articles that have been published in the last 10 years, books and periodicals on the subject in question. Individuals with Parkinson's disease under the exclusive treatment of L-dopa are more likely to develop hyperhomocysteinemia, which is a cardiovascular risk factor. Both prevention and treatment are related to a diet rich in vitamins B6, B9 and B12, acquired in a healthy and balanced diet.

Key Words: homocysteine, cardiovascular disease, Parkinson's disease, complex $b$ vitamins. 


\section{Introdução}

A doença de Parkinson (DP) é uma patologia neurodegenerativa de caráter crônico e progressivo. Descrita pela primeira vez em 1817 pelo médico inglês James Parkinson, essa enfermidade é caracterizada por uma degeneração celular na substância negra, localizada no mesencéfalo. A mesma é responsável pela produção da dopamina, um importante neurotransmissor, o qual entre outras funções, está envolvido no controle motor. Os sintomas da DP são variados, contudo, os mais comumente apresentados são a bradicinesia, rigidez muscular e o tremor em repouso (GUYTON; HALL, 2006).

O principal tratamento medicamentoso para a DP é o uso contínuo do fármaco L-dopa (prolopa) o qual atua como um precursor dopaminérgico. Essa droga tem sido associada a efeitos adversos com o uso a longo prazo, como flutuações na resposta motora, transtorno gastrointestinal e hipotensão postural, entre outros. Apesar dos efeitos colaterais, a L-dopa continua sendo o principal tratamento farmacológico na DP (O'SULLIVAN, 2010).

De acordo com Gomes (2011), a L-dopa, pode afetar o metabolismo da homocisteina, aumentando sua concentração sérica em parkinsonianos. Contudo, outros fármacos também podem ocasionar esse fenômeno, tais como: antiepilépticos, metformina, omeprazol, ciclosporina A, isoniazida e contraceptivos.

Nos últimos anos, alguns estudos têm apontado o aumento das concentrações de homocisteina (HCT) como um fator de risco cardiovascular independente e isolado. Segundo Baszczuk e Kioczyński (2014), em uma hiperhomocisteinemia (HHCT) acima de $15 \mu \mathrm{mol} / \mathrm{L}$, a cada $5 \mu \mathrm{mol} / \mathrm{L}$ aumentado, aumenta-se de $60 \%$ a $80 \%$ o risco de eventos cardiovasculares.

No Brasil, a doença cardiovascular (DCV) é responsável pela morte de pelo menos $20 \%$ da população acima de 30 anos; prevalência que aumenta ainda mais na região sul e sudeste do país (MANSUR; FAVARATO, 2016).

Em indivíduos Parkinsonianos, associação entre a HHCT com a DCV, por consequência do tratamento com a L-dopa, foi descrita pela primeira vez por Rogers et al. (2003), os quais realizaram a aferição dos níveis de homocisteína plasmática de 239 sujeitos, sendo que, 205 eram tratados com L-dopa; nesses, pôde-se observar um significativo aumento nas concentrações de HCT, cerca de $31 \%$.

Neves et al. (2004) divulgaram uma pesquisa apontando que 30\%-35\% dos indivíduos com DCV apresentaram normocolesterolemia, porém mais de $40 \%$ dos pacientes com doença primária da artéria coronária, cerebrovascular ou vascular periférica apresentaram HHCT. 
Estudos têm demonstrado que as vitaminas B6, B9 e B12 são capazes de reduzir os níveis de homocisteína séricos, podendo assim, reduzirem os riscos de desenvolvimento cardiovasculares (MARTÍ-CARVAJAL; SOLÀ; LATHYRIS, 2009).

Desse modo, o presente estudo tem por objetivo revisar na literatura o mecanismo pelo qual as vitaminas B6, B9 e B12 reduzem a HCT sérica; bem como, apresentar a fisiologia da elevação da HCT em parkinsonianos.

\section{Metodologia}

O direito Trata-se de uma revisão bibliográfica em base de dados da saúde, dentre as quais: Literatura Latino-Americana e do Caribe em Ciências da Saúde (LILACS), Scientific Eletronic Library Online (SciELO), U.S.National Libray of Medicine (PubMED), Literatura Internacional de Ciências da Saúde (MEDLINE), Centro Especializado da Organização Pan-Americana da Saúde/Organização Mundial da Saúde (BIREME) e o Google Acadêmico. Foram incluídos nessa pesquisa livros técnicos, artigos científicos e dissertações

Foram selecionados estudos que atendiam aos critérios de inclusão, são eles: terem sido publicados nos últimos 10 anos (01/05/2007 a 01/05/2018) para periódicos e, sem data limite para busca de livros os quais abordassem o tema; terem sido publicados em português, inglês ou espanhol. Os descritores utilizados foram: "doença cardiovascular", "homocisteína", "hiperhomocisteínemia", "doença de Parkinson", "tratamento com L-dopa", "doença cardiovascular" e" vitaminas do complexo B". O conectivo utilizado foi o " $\mathrm{e}$ ". Por fim, todo o material obtido foi submetido à leitura cuidadosa para a síntese e formulação do presente trabalho. Os materiais os quais não atendiam aos critérios de inclusão ou não eram referentes ao tema buscado foram descartados.

\section{Desenvolvimento}

\section{Homocisteina}

A homocisteína foi descoberta em 1932. O seu nome é devido a sua semelhança com o aminoácido cisteína (GANGULY, 2015). Segundo Venâncio et al. (2010), A HCT pode ser encontrada no organismo na forma livre (20-30\%) ou na forma associada a proteínas, de $70 \%$ a $80 \%$, e sua concentração sérica adequada varia entre 5 e $15 \mu \mathrm{mol} / \mathrm{L}$. Quando as concentrações totais se 
encontram entre $16 \mathrm{e} 30 \mu \mathrm{mol} / \mathrm{L}$, classifica-se o como HHCT moderada, entre $31 \mathrm{e}$ $100 \mu \mathrm{mol} / \mathrm{L}$ como intermediária e acima desse valor é considerada HHCT grave.

Formação e metabolismo da homocisteína

A HCT é um aminoácido sulfurado o qual não participa de síntese proteica. Ela não é obtida diretamente por meio da alimentação e sua formação se dá por meio dadesmetilação da metionina, este aminoácido sim é obtido via alimentação. No fígado, a metionina é catabolizada, dando origem a dois compostos intermediários, a S-adenosilmetionina (SAM) e posteriormente a Sadenosilhomocisteína (SAH). Após a hidrólise da SAH, forma-se adenosina e a homocisteína, a qual entra em um ciclo com duas vias alternativas, a remetilação e a transsulfuração (VENÂNCIO, et al. 2010).

$\mathrm{Na}$ remetilação atuam as enzimas metilenotetrahidrofolato redutase (MTFH) e a metionina sintase (MS), ela dá origem a metionina novamente. Uma via auxiliar na remetilação pode ser usada, nela, o grupo metil pode ser transferido da betaína, que é derivada da colina. Em ambos os casos o produto final será o mesmo, a metionina. Já na via de transulfuração, a HCT condensa-se com a serina, e há uma ação da enzima cistationina $\beta$-sintase (CBS) para produzir a cistationina 2-4, que resultará na formação da cisteína. Essa via tem por objetivo, além de participar da biossíntese da cisteína, estimular a excreção renal da HCT a fim de reduzir sua concentração plasmática (VILAÇA, et al., 2015).

Em condições fisiológicas, há um balanço equilibrado entre a produção e eliminação da HCT. As vias de metabolização da HCT utilizam as vitaminas B6, B12 e Folato como cofatores (VANNUCCHI; MELO, 2009).

A HCT pode seguir outros caminhos ao ser metabolizada além das vias de remetilação e transulfuração. Em um dos caminhos, ocorre oxidação, em que duas moléculas de HCT podem se associar e dar origem a homocistina. Outra alternativa é a HCT formar a homocisteína tiolactona, por meio da perda de uma molécula de água. Sua auto-oxidação também pode dar origem a radicais livres, como o peróxido, superóxido de hidrogênio e radical hidroxila, os quais são moléculas reativas de oxigênio e são consideradas substâncias tóxicas ao organismo humano (BASZCZUK; KOPCZYNSKI, 2014).

Homocisteína como um marcador de risco cardiovascular

Evidências sugerem que a HHCT representa um importante marcador biológico independente para DCV, por aumentar os níveis de substâncias próoxidantes favorecendo a oxidação do LDL-colesterol, em especial, doença vascular aterosclerótica e trombose (GONZÁLEZ-GROSS et al., 2012)

A HCT aumentaria os níveis de dimetilarginina assimétrica (ADMA), o qual é um inibidor do óxido nítrico sintase (eNOS), enzima atuante na produção do óxido nítrico (NO); o qual, desenvolve função vaso dilatadora e impede a adesão plaquetária no endotélio vascular. Por consequência da anulação 
enzimática, o óxido nítrico sintase e o óxido nítrico seria suprimido. Acredita-se que esse mecanismo cause danosno endotélio vascular e alterações na coagulação plaquetária, levando assim, a complicações cardiovasculares em artérias cerebrais, coronárias e periféricas (SHENOV et al., 2014).

Segundo Vannucchil e Melo (2009), a HHCT está envolvida com danos no sistema cardiovascular, tais como a injúria causada no endotélio vascular, hiperplasia celular na musculatura lisa vascular, maior adesividade plaquetária, aumento da oxidação do LDL-colesterol com deposição na parede vascular, fibrose e calcificação das placas de ateroma e ativação direta da cascata de coagulação. Ainda segundo o autor, devido ao efeito tóxico da homocisteína tiolactona, há alterações no endotélio vascular, pois parte da HCT é auto-oxidada e forma radicais livres, causando lesão celular no endotélio, o que causa a ativação da cascata de coagulação, levando a um quadro de trombose.

De 5\% a 7\% da população mundial apresenta HHCT, e 47\% dos indivíduos com doença vascular aterosclerótica sintomática apresentam HHCT moderada (VENANCIO et al., 2010).

Apesar de bem estabelecida como um biomarcador de DCV, ainda não está bem elucidado o mecanismo fisiopatológico pelo qual a HHCT atua na gênese da DCV, todavia, o estresse oxidativo parece ser um fator determinante (PINTO et al., 2009).

Hiperhomocisteinemia gerada pela L-dopa na doença de Parkinson

Günaydın et al. (2016) realizaram um estudo transversal na Turquia para avaliar o risco cardiovascular em doentes com a enfermidade de Parkinson. Foram selecionados 65 sujeitos os quais utilizavam exclusivamente o fármaco Ldopa. Indivíduos com outras morbidades associadas ao Parkinson foram excluídos. O estudo ainda contou com um grupo controle de 32 indivíduos saudáveis. Os sujeitos foram acompanhados por 19 meses. Após esse período, por meio de exames, notou-se que os pacientes com a DP apresentavam uma rigidez aórtica e um prejuízo da função diastólica, também foram observados níveis elevados de HCT plasmática quando comparados a pessoas saudáveis do grupo controle. Análises estatísticas apontaram que os resultados foram significativos.

Uma meta-análise, desenvolvida na China, foi realizada a fim de verificar na literatura a associação entre a L-dopa e a HHCT. Para isso, recorreu-se às técnicas estatísticas para revelar se há uma correlação significante. O estudo conclui relatando que o tratamento da DP com a L-dopa está associado a um aumento dos níveis séricos de HCT sérica (HU et al., 2013)

Segundo Ferreira (2013, p. 2) 


\begin{abstract}
O grande destino metabólico da L-dopa plasmática envolve a sua o-metilação para formar a 3-O-metildopa, uma reação que é catalisada pela enzima catecol-O-metiltransferasee onde o sadenosilmetionina age como um doador de grupo metilo. $\mathrm{O}$ catabolismo da L-dopa pode, portanto, interferir em vários níveis com o metabolismo da homocisteína, aumentando os seus níveis plasmáticos.
\end{abstract}

Sendo assim, por meio do metabolismo da L-dopa, dá-se a formação da HCT, elevando-a no plasma.

Ibrahimagic et al. (2016) estimam que 1 a cada 5 pacientes $(20 \%)$ com a DP desenvolve certo grau de HHCT. Para Belcastro et al. (2010), 20\% a 30\% dos indivíduos com a DP apresentam níveis séricos de HCT elevados. Os níveis de HCT elevados em sujeitos com a DP, parece ser consequência do uso prolongado do fármaco L-dopa, utilizado em seu tratamento, e não por consequência da própria doença (SHIN; SOHN, 2009). Entretanto, segundo o trabalho de Müller. et al (2009). citados por Ferreira, (2013) e Ibrahimagic et al. (2016), fármacos da classe inibidores da enzima catecol-O-metil transferase (entacapona, tolcapona e tropolona), quando associados à L-dopa no tratamento da DP, não permitem que a homocisteína se eleve a níveis maiores do que quando a L-dopa é o único fármaco utilizado.

Segundo Vilaça et al. (2015), além do risco cardiovascular, o aumento nos níveis de HCT provoca sensibilização de neurônios dopaminérgicos a danos induzidos por íons de ferro na substância negra do mesencéfalo, podendo acelerar a evolução da DP.

Não foram encontrados estudos referentes ao índice de mortalidade de indivíduos Parkinsonianos com HHCT.

Hiperhomocisteinemia vitaminas B6, B12 e folato

Concentrações de homocisteína acima da recomendada, isto é, até 15 $\mu \mathrm{mol} / \mathrm{L}$ são consideradas um fator de risco para DCV. As concentrações de homocisteína no plasma podem se elevar com a deficiência de vitaminas do complexo B, mais precisamente as vitaminas B6, B12 e o folato (FERREIRA, 2013; ANSARI et al., 2014; IBRAHIMAGIC et al., 2016). Todavia, para Collin et al. (2010) citados por Steluti (2010), deve-se levar em conta que outros fatores são capazes de influenciar no metabolismo e nas concentrações de HCT, tais como: defeitos genéticos, doenças (anemia perniciosa, problemas renais, hipotireoidismo, psoríases etc.) e alguns medicamentos antagonistas de folato e vitamina B6. 
Segundo Venâncio et al. (2010), a formação da 5-metilenotetrahidrofolato (5-MTHF), forma ativa do folato no plasma, seria afetada pela baixa ingesta de folato dietético, o que influenciaria na remetilação da HCT. Da mesma forma, a deficiência dietética de vitamina B12 comprometeria a remetilação da HCT, não por insuficiência da 5-MTHF, mas sim por um prejuízo natransferência em seu radical metil para a $\mathrm{HCT}$, por meio da via de remetilação. Já a insuficiência de vitamina B6 afetaria, por meio da via de transulfuração, a conversão de HCT no aminoácido cisteína, pois, as enzimas responsáveis por tal processo (CBS e ycistationase) são ativadas pela piridoxina. Logo, a deficiência dietética dessas vitaminas resultaria no acúmulo da HCT intracelular, em seguida ela seria transportada para o meio extracelular, o que causaria a elevação sérica de HCT. Ainda segundo o autor, a suplementação vitamínica de ácido fólico, B6 e B12 tem se mostrado um eficiente no controle da HHCT.

Segundo Dantas, Frank e Soares (2008) tem sido proposto o enriquecimento da dieta com vitaminas do complexo B, para aumentar o catabolismo da homocisteÌna e diminuir a hiperhomocisteinemia e suas consequências em pacientes com a DP tratados com a L-dopa.

A ingestão extra das vitaminas B6, B9 e B12, com o objetivo de reduzir o risco cardiovascular, ainda é controversa. Shin; Sohn, (2009), verificaram os níveis séricos de HCT total de 74 indivíduos, dentre os quais, 33 possuíam a DP, os outros 41 eram saudáveis. Após a análise bioquímica, foi constatado que os indivíduos com a DP apresentavam níveis de HCT mais elevados quando comparados ao grupo controle. Segundo os autores a suplementação de vitaminas B6, B12 e folato é indicada para pacientes com HHCT.

Um estudo observacional retrospectivo foi realizado no Hospital de S. João E.P.E. pelo período de 2002 a 2012, com 55 sujeitos com a DP (média de idade 67 anos) como grupo experimental e 55 saudáveis, com as idades semelhantes, como grupo controle. O estudo foi realizado com o objetivo de verificar as concentrações séricas de Homocisteína total, folato e vitamina B12. Os indivíduos utilizavam a L-dopa como principal tratamento, apesar de utilizarem outros fármacos associados. A dose diária média de L-dopa era de 823,53 mg/dia ( \pm 406,04 mg).

Após o acompanhamento, chegou-se à conclusão que os indivíduos parkinsonianos apresentavam uma concentração sérica de folato ligeiramente reduzida, quando comparada com o grupo controle. Já os níveis de vitamina B12 estavam significativamente mais reduzidos nos sujeitos com a DP. Inversamente, os níveis de HCT estavam 61,1\% mais elevados no grupo com a DP. Em indivíduos parkinsonianos os quais utilizavam o fármaco entacapona associado à L-dopa (23), apesar de apresentarem baixa concentração de folato e vitamina B12, os níveis de HCT estavam mais reduzidos. $\mathrm{O}$ autor sugere que uma 
terapêutica complementar com folato e vitamina B12 deve ser utilizada em indivíduos com a DP a fim de normalizar os níveis das vitaminas e da HCT, de forma a atrasar as consequências ao longo da evolução da DP (FERREIRA, 2013).

DiFrancisco-Donoghu et al. (2012), realizou um ensaio randomizado com 36 indivíduos com a DP e com HHCT, isto é, níveis de HCT acima de $15 \mu \mathrm{mol} / \mathrm{L}$. Os indivíduos foram divididos em grupos. Um dos grupos recebeu uma suplementação de vitamina B6, B12 e folato, duas vezes por dia durante 6 semanas. Após o período de intervenções, chegou-se ao resultado de que a suplementação vitamínica reduziu a concentração sérica de HCT nos indivíduos com a DP.

Já a Sociedade Brasileira de Cardiologia, por meio da I Diretriz e Prevenção Cardiovascular, não recomenda a suplementação dessas vitaminas com o objetivo de reduzir o risco cardiovascular, pois segundo a mesma, estudos prospectivos randomizados com grande número de eventos cardiovasculares falharam em mostrar benefício da suplementação de folato e complexo B com intuito de reduzir a homocisteína e prevenção da DCV (SIMÃO et al., 2013, p. 13).

Uma revisão foi efetuada com o objetivo de avaliar o impacto da intervenção, com suplementos vitamínicos a base de vitamina B6, B9 e B12, na HHCT sob o ponto de vista de prevenção de eventos cardiovasculares. Após a revisão e análise estatística, o estudo conclui que não há evidência suficiente para a utilização de suplementos com as vitaminas B6, B9 e B12 com o intuito de evitar eventos cardiovasculares (MARTÍ-CARVAJAL; SOLÀ; LATHYRIS, 2009).

\section{Considerações Finais}

Assim, Indivíduos com a DP, sob o tratamento com a droga L-dopa, têm níveis de HCT mais elevados do que indivíduos saudáveis. Isso devido à formação da HCT decorrente do metabolismo da droga. Todavia, esses níveis são um pouco menores quando a farmacoterapia para a DP envolve outros medicamentos da classe inibidores da enzima catecol-O-metil transferase associados a L-dopa.

A HHCT é caracterizada como níveis séricos de HCT acima de $15 \mu \mathrm{mol} / \mathrm{L}$. Ela traz consigo diversas consequências, dentre elas está o potencial risco de eventos cardiovasculares, isso devido ativação de substâncias pró-inflamatórias que participam da oxidação do LDL-colesterol. O risco cardiovascular da HHCT é potencializado a cada $5 \mu \mathrm{mol} / \mathrm{L}$ de HCT aumentados no sangue.

A HHCT está inversamente relacionada com os níveis séricos das vitaminas B6, B9 e B12. Até o presente momento, faltam evidências científicas 
para recomendar a suplementação de tais vitaminas a fim de reduzir o risco cardiovascular nos mesmos. Contudo, uma alimentação que atenda às recomendações diárias das vitaminas B6, B12 e folato, mostra-se eficaz na prevenção e no controle da HHCT, logo, indivíduos com a DP devem garantir a ingestão diária destas vitaminas de forma adequada a fim de prevenir um quadro de HHCT e suas consequências.

Salienta-se que mais estudos devem ser realizados a fim de elucidar os efeitos das vitaminas B6, B9 e B12, sobre a HHCT e suas consequências, em indivíduos com a DP.

\section{Referências}

ANSARI, R. et al. Hyperhomocysteinemia and neurologic disorders: A review. Journal of Clinical Neurology. (Seul, Coréia), v.10, n.4, p.281-288, 2014.

BASZCZUK, A.; KIOCZYNSKI, Z. Hyperhomocisteynemia in patients with cardiovascular disease. Postępy Higieny i Medycyny Doświadczalnej (Online); v. 68, p.579-589, 2014.

BELCASTRO, V. et al. Hyperhomocysteinemia recurrence in L-dopa-treated Parkinson's disease patients. European Journal of Neurology. v.17, n5, p.661 $665 ; 2010$.

COLLIN, S.M., et al. Circulating folate, vitamin B12, homocysteine, vitamin B12 transport proteins, and risk of prostate cancer: a case-control study, systematic review, and meta-analysis. Cancer Epidemiology, Biomarkers \& Prevention, v. 10, p. 1632-42, 2010.

DANTAS, A.M.C; FRANK, A.A.; SOARES, E.A. Vitaminas antioxidantes na Doença de Parkinson. Revista Brasileira de Geriatria e Gerontologia. v. 11, n. 1, p. 105-116, 2008.

DIFRANCISCO-DONOGHUE, J. et al. Effects of exercise and B vitamins on homocysteine and glutathione in Parkinson's disease: a randomized trial. Neurodegenerative Diseases. v.104, n.4, p.127-134; 2012. 
FERREIRA, M. I. Níveis séricos de folato e vitamina B12 em doentes de Parkinson tratados com L-dopa. Dissertação (Mestrado em nutrição clínica) Universidade do Porto; 2013.

GANGULY, P.; ALAM, S.F. Role of homocysteine in the development of cardiovascular disease. Nutrition Journal. v.14, n.6, p.3-16, 2015

GOMES, J. R. P. Acidente vascular cerebral e homocisteinemia. Dissertação de Mestrado, Universidade do Porto, 2012.

GONZÁLEZ-GROSS, M. et al. Gender and age influence blood folate, vitamin B12, vitamin B6, and homocysteine levels in European adolescents: the Helena Study. Nutrition Research New York, v.32, n.11, p.817- 826, 2012.

GÜNAYDIN, Z.Y; et al. Evaluation of cardiovascular risk in patients with Parkinson disease under L-dopa treatment. Journal of Geriatric Cardiology v. 13, n.1, p.75-80. 2016.

GUYTON, A.C.; HALL, J.E. Tratado de Fisiologia Médica. 11aㅗ ed. Rio de Janeiro, Elsevier Ed., 2006.

HU, X.W. et al. Elevated homocysteine levels in L-dopa-treated idiopathic Parkinson's disease: A meta-analysis. Acta Neurologica Scandinavica; v128, n.2, p.73-82.; 2013.

IBRAHIMAGIC, O.C. et al. Hyperhomocysteinemia and Its Treatment in Patients with Parkinson's Disease. Mater Sociomedic v.28, n4, p.303-306. 2016.

MANSUR AP, FAVARATO D. Tendências da Taxa de Mortalidade por Doenças Cardiovasculares no Brasil, 1980-2012. Arquivo Brasileiro de Cardiologia, 2016.

MARTI-CARVAJAL, A. J. et al. Homocysteine lowering interventions for preventing cardiovascular events. Cochrane Database Syst Rev. 2009. 
MÜLLER, T.; MUHLACK, S. Peripheral COMT inhibition prevent L-dopa associated homocysteine increase. Journal of Neural Transmission, Vol. 116:1253-1256; 2009.

NEVES L.B., MACEDO D.M., LOPES A.C. Homocisteína. Jornal Brasileiro de Patologia e Medicina Laboratorial; v.40, n. 5, p. 311-20, 2004.

O' SULLIVAN, S. B.; SCHMITZ, THOMAS J. Fisioterapia Avaliação e Tratamento. $5^{\circ}$ ed. São Paulo: Manole, 2010.

PINTO, W.J. et al. Homocisteína e risco cardiovascular. Revista de Ciências Médicas-ISSN 2318-0897, v. 18, n. 5/6, 2009.

ROGERS JD, et al. Elevated plasma homocysteine levels in patients treated with L-dopa: association with vascular disease. Jama Neurology. v. 60, n. 1, p. 59-64; 2003.

SHENOV V, et al. Correlation of serum homocysteine levels with he severity of coronary artery disease. Indian Journal of Clinical Biochemistry, v. 29, n. 3, p.339442014.

SHIN, H.W.; SOHN, Y.H. Hyperhomocysteinemia in patients with Parkinson's disease and relationship to vitamin B level. Journal of Movement Disorders, v. 2, p. 33-36, 2009.

SIMÃO, A.F. et al. Sociedade Brasileira de Cardiologia. I Diretriz Brasileira de Prevenção Cardiovascular. Arquivos Brasileiros de Cardiologia 101 (6 Supl.2): S1- 63; 2013.

STELUTI, J. et al. Folato, B6 e B12 na adolescência: níveis séricos, prevalência de inadequação de ingestão e alimentos contribuintes. Journal of Pediatric (Rio J.) [online], Porto Alegre, v. 87, n. 1, p. 43-49; Feb. 2011.

VANNUCCHI, H.; MELO, S. hiperhomocisteinemia e risco cardiometabólico. Arquivo Brasileiro de Endocrinologia e Metabologia, São Paulo, v. 53, n. 5, p. 540$549,2009$. 
ALVES, J.N.C.;JUNIOR,F.A.C.S.; SANTOS, J.M.. Imunização em crianças infectadas pelo HIV: uma leitura de cobertura vacinal para pneumocócica 23v R. Científica UBM - Barra Mansa (RJ),

ano XXIII, v. 20, n. 39, 2. Sem. 2018. págs. 26-38.

ISSN 1516-4071

VENANCIO, L.; BURINI, R.C.; YOSHIDA, W.B. Tratamento dietético da hiperhomocisteinemia na doença arterial periférica. Jornal Vascular Brasileiro. Porto Alegre, v. 9, n. 1, p. 28-41, 2010.

VILAÇA et al. Metabolismo da homocisteína em doenças neurológicas. Revista Brasileira de Neurologia, [S.1.], v. 51, n.3, p.73-78. 2015. 